\title{
A family of 2 mirror unobscured wide field telescope and collimator designs (Withdrawal Notice)
}

Richard Horton

Richard F. Horton, "A family of 2 mirror unobscured wide field telescope and collimator designs (Withdrawal Notice)," Proc. SPIE 7652, International Optical Design Conference 2010, 76520M (4 February 2011); doi: $10.1117 / 12.871072$

SPIE Event: International Optical Design Conference 2010, 2010, Jackson Hole, WY, United States 


\section{A family of 2 mirror unobscured wide field telescope and collimator designs (Withdrawal Notice)}

Richard F. Horton

Ad hoc Optics LLC (China)

Proceedings Volume 7652, International Optical Design Conference 2010; $76520 \mathrm{M}$ (2011)

https://doi.org/10.1117/12.871072

Event: International Optical Design Conference 2010, 2010, Jackson Hole, WY, United States

Online Publication Date: 4 February 2011

Withdrawn from Publication: 9 August 2019

Publisher's Note: This paper was published in error and has been withdrawn. A correct version of the paper can be found at https://doi.org/10.1117/12.857310. The Publisher regrets the error. 